\title{
Dextrocardia con doble salida del ventrículo derecho con comunicación interventricular no relacionada asociada a malposición de grandes vasos
}

\section{Right ventricular double outlet dextrocardia with unrelated ventricular septal defect associated with great vessel malposition}

\author{
Giovanny Ríos-Dueñas ${ }^{1,2 *}$, José A. Galán-Cadena ${ }^{2}$, Natalia Olmos-Muskus ${ }^{2}$ e Isabella Rivera-Tobar ${ }^{2}$ \\ ${ }^{1}$ Servicio de Cirugía Cardiovascular, Hospital Universitario San Ignacio; ${ }^{2}$ Facultad de Medicina, Pontificia Universidad Javeriana. Bogotá, Colombia
}

\section{Resumen}

En el siguiente reporte se expone un caso complejo en el que coexisten malformaciones cardiacas congénitas de presentación atípica con un doble tracto de salida del ventrículo derecho, en un paciente con múltiples anomalías menores en la exploración física y un cariotipo normal. La atipia de cada una de estas y su coexistencia hacen pensar en posibles alteraciones genéticas que aún son desconocidas. Lo anterior supone un reto terapéutico con el fin de restaurar una fisiología cardiaca compatible con la vida, lo cual se logra en este caso mediante un cerclaje exitoso de la arteria pulmonar.

Palabras clave: Doble salida del ventrículo derecho. Dextrocardia. Hipertensión pulmonar. Arteria pulmonar.

\section{Abstract}

The following report presents a complex case in which congenital cardiac malformations of atypical presentation coexist with a double outlet right ventricle, in a patient with multiple minor abnormalities on the physical examination and a normal karyotype. The atypia of these and their coexistence suggest possible genetic alterations that are still unknown. Therefore, a therapeutic challenge in order to restore a cardiac physiology compatible with life is proposed, which is achieved in this case through a successful banding of the pulmonary artery.

Key words: Double outlet right ventricle. Dextrocardia. Pulmonary hypertension. Pulmonary artery.

\section{Introducción}

La prevalencia de las cardiopatías congénitas en Bogotá es de 15.1 por 10.000 nacidos vivos según un estudio realizado en el periodo entre 2001 y 2014, el cual mostró valores superiores durante los últimos 3 años de seguimiento (20 por 10.000 nacidos vivos). En total, el $2.03 \%$ de los nacimientos tuvieron algún tipo de malformación y el $12.07 \%$ corresponden a cardiopatías complejas ${ }^{1}$.

El doble tracto de salida del ventrículo derecho (DSVD) es una cardiopatía de origen conal que se define como una conexión ventriculoarterial en la cual las

\section{Correspondencia:}

*Giovanny Ríos-Dueñas

E-mail: edgar.rios@javeriana.edu.co
Fecha de recepción: 10-04-2020

Fecha de aceptación: 21-04-2021 DOI: 10.24875/RCCAR.M21000070
Disponible en internet: 18-10-2021 Rev Colomb Cardiol. 2021;28(4):383-388 www.rccardiologia.com 0120-5633 / @ 2021 Sociedad Colombiana de Cardiología y Cirugía Cardiovascular. Publicado por Permanyer. Este es un artículo open access bajo la licencia CC BY-NC-ND (http://creativecommons.org/licenses/by-nc-nd/4.0/). 
grandes arterias emergen totalmente 0 de manera predominante del ventrículo anatómicamente derecho ${ }^{2}$. La coexistencia de DSVD, comunicación interventricular (CIV) no relacionada y malposición de grandes vasos es infrecuente; produce una alteración en la fisiología cardiaca usual y supone un desafío quirúrgico y clínico. El objetivo de este reporte es mostrar el abordaje terapéutico de un caso de cardiopatía congénita atípica sin alteración genética aparente que fue llevado a un cerclaje pulmonar con el fin de restablecer una fisiología cardiaca compatible con la vida.

\section{Caso clínico}

\section{Prequirúrgico}

Paciente de 4 meses, perteneciente a una comunidad indígena colombiana, fruto de primera gestación de binomio sin relación consanguínea aparente, con escasos controles prenatales y parto domiciliario. Cuenta con familiares en segundo grado de consanguinidad con cardiopatía no clara, en quienes se ha hecho necesaria intervención quirúrgica.

Acude a los 3 meses de edad a un centro de atención primaria con cuadro respiratorio agudo, donde se documenta un soplo holosistólico IV/VI y se le realiza un ecocardiograma que reporta cardiopatía compleja. Por lo anterior, se remite a una institución de cuarto nivel donde se inicia antibioticoterapia y soporte nutricional por desnutrición grave asociada. Se realiza un ecocardiograma que evidencia situs solitus en dextrocardia, doble salida del ventrículo derecho con vasos malpuestos, con comunicación interventricular (CIV) del tracto de entrada grande de $8 \mathrm{~mm}$ no relacionada, ventrículo derecho hipoplásico con overriding de la tricúspide, hipertensión pulmonar grave, cavidades izquierdas dilatadas con signos de sobrecarga de volumen y función ventricular izquierda conservada (fracción de eyección del 70\%) (Fig. 1).

Ante este hallazgo y la necesidad de medir variables hemodinámicas, se realiza cateterismo cardiaco que evidencia fisiología univentricular de hiperflujo sin estenosis pulmonar, hipertensión pulmonar grave hipercinética 3 unidades Wood y resistencias pulmonares móviles y bajas al test de hiperoxia (Figs. 2-3). Adicionalmente muestra el seno coronario drenando solo a vena vertical e innominada. Por lo anterior, se consideró que el paciente no era candidato a cirugía correctora biventricular y se decide llevar a una primera aproximación quirúrgica con cerclaje de la arteria pulmonar.

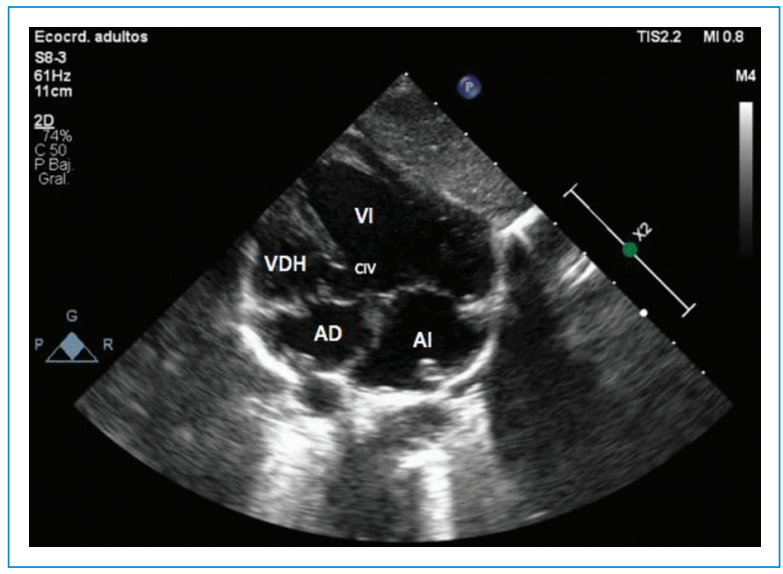

Figura 1. Dextrocardia con situs solitus asociado a ventrículo derecho hipoplásico con una comunicación interventricular (CIV) no relacionada y primordial de la válvula tricúspide. $\mathrm{AD}$ : aurícula derecha; Al: aurícula izquierda; VDH: ventrículo derecho hipoplásico; VI: ventrículo izquierdo.

Fue valorado por el servicio de genética médica, quienes encontraron en la exploración física múltiples anomalías menores, como turricefalia, telecanto, lóbulos auriculares adheridos, antihélix prominente, cuello corto, retrognatia e hipertelorismo mamario. Por lo anterior, se solicita un cariotipo con bandeo G, cuyo resultado es normal.

\section{Intraquirúrgico}

El paciente fue llevado a cirugía y se encontró el tronco de la arteria pulmonar de ubicación derecha y posterior, y se midió de forma directa una presión pulmonar prebandeo de 58/14 mmHg. Se realizó cerclaje con una banda de un segmento de un injerto de dacrón de $8 \mathrm{~mm}$, con la cual se hicieron múltiples pruebas progresivas de oclusión con hemoclips hasta lograr un aumento de $10 \mathrm{mmHg}$ de la presión sistólica y una reducción del $15 \%$ de la saturación de oxígeno. Se repitió la medición directa de la presión pulmonar posbandeo, que fue de $33 / 13 \mathrm{mmHg}$, y se fijó el segmento de injerto con puntos separados de monofilamento no absorbible 6-0 (Fig. 4). Se terminó el procedimiento sin complicaciones y el paciente fue llevado a la unidad de cuidado intensivo pediátrico.

\section{Posquirúrgico}

El paciente continúa con ventilación mecánica, con saturaciones entre el $75 \%$ y el $85 \%$, y bajo 


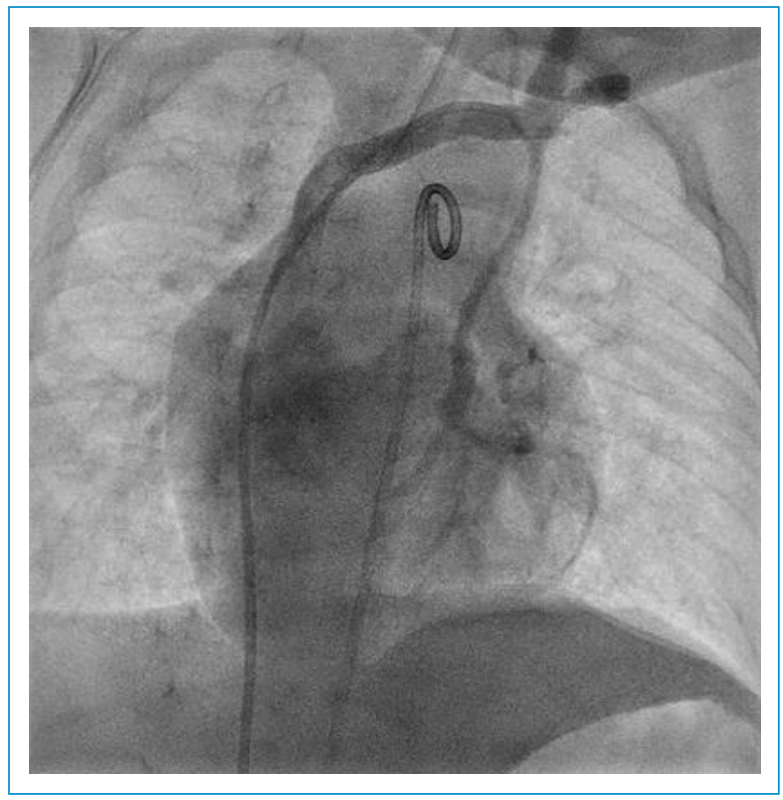

Figura 2. Cateterismo cardiaco que evidencia una vía venosa llegando a la vena cava superior izquierda que se conecta con el seno coronario, el cual termina en fondo ciego, por lo que drena retrógradamente por la vena cava superior izquierda a una vena innominada.

sedoanalgesia para disminuir el consumo de oxígeno $\left(\mathrm{VO}_{2}\right)$. Durante el posoperatorio cursó con episodios de desaturación, hipotensión e hipoperfusión relacionadas al despertar sugestivas de crisis de hipertensión pulmonar, por lo que se inició soporte inotrópico, vasopresor, balanceo de circulación y transfusión de glóbulos rojos debido a anemia (hemoglobina: 8.9), con lo que presentó una mejoría de su estado hemodinámico. Posteriormente el paciente tolera el descenso progresivo de la fracción inspiratoria de oxígeno en el aire inspirado $\left(\mathrm{FiO}_{2}\right)$ y se intenta realizar la extubación, pero no la tolera por crisis de hipertensión pulmonar asociada a bradicardia y pobre esfuerzo respiratorio, por lo que requiere nuevamente soporte ventilatorio invasivo. Se realizó un ecocardiograma (Fig. 5) que reportó disfunción sistólica con fracción de eyección del 50\% y un gradiente a través del bandeo protector de $40 \mathrm{mmHg}$, ante lo cual se instauró manejo antifalla con enalapril y furosemida. El paciente presenta una evolución clínica favorable y se realiza extubación programada, manteniendo las saturaciones de oxígeno esperadas por su patología con cánula nasal de alto flujo y luego con cánula nasal convencional, con adecuada tolerancia a la

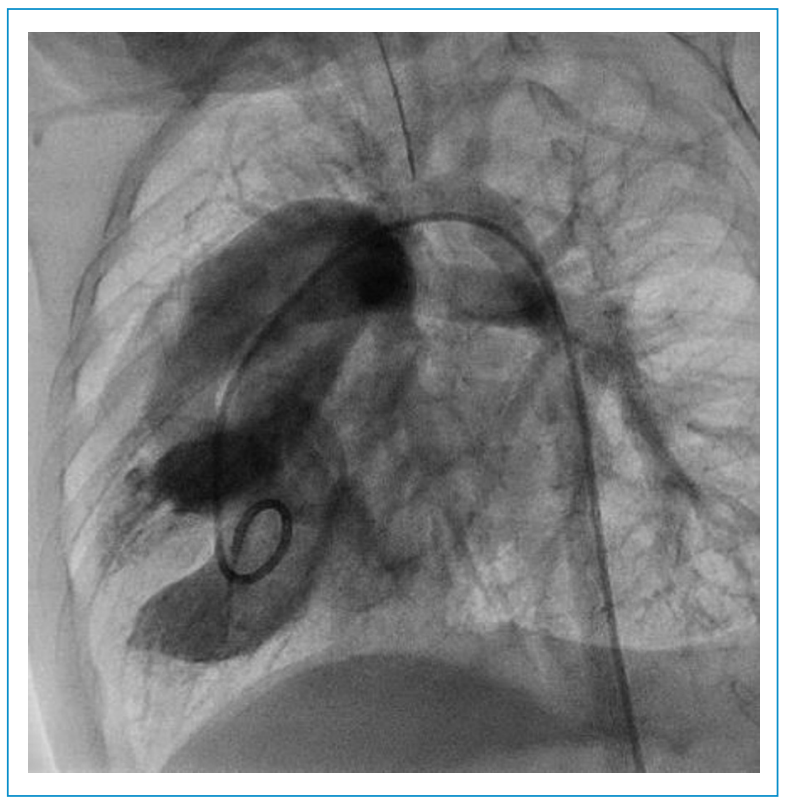

Figura 3. Cateterismo cardiaco que evidencia un ventrículo posterior e inferior de morfología izquierda y cámara rudimentaria anterior y superior de morfología derecha, donde emergen los vasos, estando la pulmonar derecha marcadamente dilatada y la aorta izquierda y anterior.

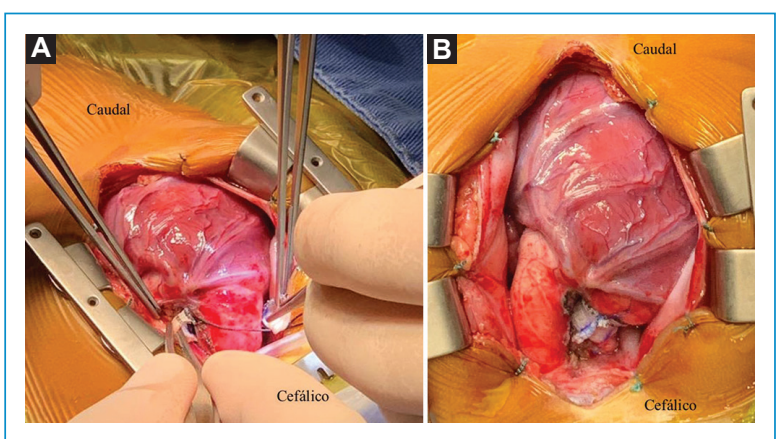

Figura 4. A: paso de banda de un segmento de un injerto de dacrón Gelsoft Plus de $8 \mathrm{~mm}$ en el tronco de la arteria pulmonar. B: cerclaje de la arteria pulmonar.

suspensión de soportes, por lo que es trasladado a hospitalización en piso.

Durante su estancia se indicó manejo conjunto con nutrición, fonoaudiología, psicología y terapia física y ocupacional, con el fin de brindar una rehabilitación integral del paciente crítico. Además, trabajo social inició los trámites administrativos de remisión a una institución de salud para continuar el manejo intrahospitalario cerca de su lugar de procedencia. El paciente cursó con aumento del 


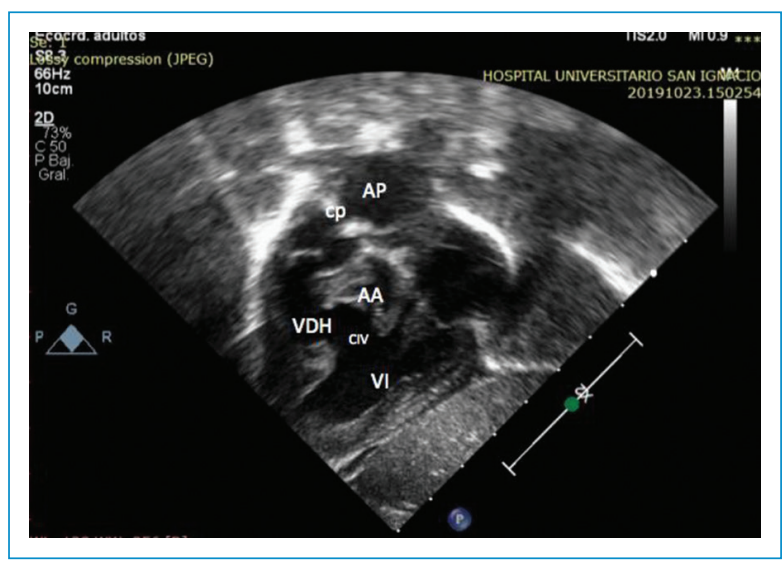

Figura 5. Doble tracto de salida del ventrículo derecho hipoplásico; adicionalmente, se evidencia el cerclaje de la arteria pulmonar. AA: arteria aorta; AP: arteria pulmonar; CIV: comunicación interventricular; $c p$ : cerclaje de la arteria pulmonar; VDH: ventrículo derecho hipoplásico; VI: ventrículo izquierdo.

esfuerzo respiratorio secundario a sobrecarga hídrica, por lo que se decidió ajustar la dosis de furosemida, con posterior resolución. Finalmente, el paciente presentó una evolución hacia la mejoría hasta que se logró la remisión al hospital en su lugar de origen. Se dio egreso con manejo antifalla, profilaxis antibiótica para endocarditis y analgesia. También se entregaron órdenes para cita de control con cardiología pediátrica y cirugía cardiovascular. Se estableció un plan de controles en su lugar de origen y una vez complete el periodo estimado para un segundo tiempo quirúrgico (cirugía de Glenn) se ordenó remitir.

\section{Discusión}

EI DSVD representa un complejo espectro de polimorfismos anatómicos con distintas manifestaciones clínicas y abordajes quirúrgicos. Existen diversas clasificaciones, pero la más utilizada es la descrita por Lev, et al..$^{3}$, que consta de cuatro tipos de acuerdo con el defecto del tabique ventricular en relación con las grandes arterias.

El primer tipo es el DSVD con CIV subaórtica, que es el más frecuente y se presenta hasta en un $50 \%$ de los casos $^{2}$. Puede comportarse como una fisiología de CIV aislada, en caso de no tener obstrucción del tracto de salida pulmonar, o con una fisiología de tetralogía de Fallot en caso de tenerla.
El segundo tipo es el DSVD con CIV subpulmonar, también llamado malformación de Taussig-Bing cuando no tiene obstrucción del tracto de salida pulmonar $^{2}$, el cual se reporta hasta en un $30 \%$ en las series quirúrgicas de DSVD $^{4}$ y presenta una fisiología de transposición de grandes vasos, ya que la posición de la CIV favorece el flujo del ventrículo izquierdo hacia la circulación pulmonar. El tercer tipo es el DSVD con CIV doblemente relacionada, en la que el defecto del tabique se encuentra superior a la trabécula septomarginal y debajo de las valvas semilunares $^{2}$. Ocurre hasta en un $10 \%$ de los pacientes con DSVD y debido a la ausencia de tabique interventricular la sangre fluirá libremente a las arterias aorta y pulmonar. Por último está el DSVD con CIV no relacionada, que corresponde a un defecto del tabique ventricular inusual, en general posterior, que se encuentra a una distancia considerable de las válvulas pulmonar y aórtica ${ }^{5}$.

Adicionalmente, el DSVD también se ha clasificado según la relación entre las grandes arterias en tres grupos: grandes arterias ligeramente entrecruzadas, paralelas en el plano frontal (lado a lado) y con aorta anterior y arteria pulmonar posterior; este último grupo tiene a su vez tres variantes anatómicas: aorta anterior derecha con arteria pulmonar posterior izquierda, aorta anterior con pulmonar posterior y aorta anterior izquierda con pulmonar posterior derecha, siendo esta última la malposición de grandes vasos que presenta el paciente descrito, la cual se ha reportado en un $3.3 \%$ en las series de $\operatorname{casos}^{6}$.

Por otra parte, la dextrocardia y la hipoplasia del ventrículo derecho son otras alteraciones que se han reportado de forma atípica en algunos casos descritos en la literatura ${ }^{7}$, que se asocian en este caso a un tipo poco frecuente de DSVD.

Con respecto al manejo, dependiendo de la relación de las grandes arterias, la obstrucción del tracto de salida del ventrículo derecho y la disposición del defecto septal frente a la aorta, se evalúa la posibilidad de crear un túnel intracardiaco protésico para hacer una reparación biventricular. Sin embargo, en algunos casos, como el aquí descrito, la localización de la CIV con respecto a la válvula aórtica imposibilita esta opción, por lo que es necesario recurrir a una fisiología distinta que permita ser compatible con la vida, como puede ser una reparación univentricular con las cirugías de Glenn y Fontan, respectivamente. 
La hipertensión pulmonar hipercinética se asocia al DSVD secundario a un hiperflujo pulmonar por la diferencia de resistencias entre las circulaciones pulmonar y sistémica ${ }^{8}$, lo que puede generar desenlaces negativos en la cirugía de Fontan ${ }^{9}$. Por esta razón, en el presente caso se optó por un manejo paliativo univentricular iniciando con un cerclaje de arteria pulmonar para limitar el flujo sanguíneo y evitar el desarrollo de una enfermedad vascular y de disfunción ventricular por sobrecarga crónica de volumen, que conllevan pobres resultados quirúrgicos en las cirugías de fisiología univentricular ${ }^{9}$.

El cerclaje de arteria pulmonar se ha asociado a diversas complicaciones, como tensión insuficiente de la banda que favorece su migración y genera estenosis de las ramas de la arteria pulmonar, sobre todo la derecha porque surge más proximal al tronco arterial y, por tanto, tiene mayor riesgo de obstrucción ${ }^{10}$. También se ha asociado a erosión del lumen del vaso con posterior dilatación del tronco pulmonar y fragilidad de la pared arterial, predisponiendo a la formación de pseudoaneurismas ${ }^{11}$. Además, puede generar insuficiencia pulmonar secundaria a la dilatación del anillo pulmonar 0 a la distorsión de las valvas ${ }^{10}$. Sin embargo, en este caso fue posible llevar a cabo un tratamiento individualizado de forma exitosa, obteniendo un adecuado gradiente a través del cerclaje que proporcionará al paciente una calidad de vida aceptable hasta que sea llevado a la corrección quirúrgica definitiva ${ }^{12}$.

Por otro lado, en diversos estudios se ha identificado que alrededor del $30 \%$ de los pacientes con cardiopatía congénita presentan un síndrome genético 0 una asociación con anomalías cardiacas o extracardiacas $^{13}$. Sin embargo, la coexistencia de múltiples anomalías encontradas en el presente caso no corresponde a un síndrome genético conocido.

La frecuencia del diagnóstico genético en fetos con cardiopatías congénitas graves fue estimada en un estudio reciente en el que, después de excluir los casos con aneuploidías, se logró realizar en un $15.7 \%$ de los casos $^{14}$. En los países desarrollados, el análisis de paneles de genes en el cuidado estándar del feto permite alcanzar estas cifras, pero en nuestro medio, en casos como el presente, el diagnóstico queda incierto por la falta de recursos para realizar estas pruebas. A pesar de contar con un cariotipo normal, hacer un panel de genes habría permitido explorar alteraciones genéticas específicas. Ejemplo de ello es el gen PITX2, conocido como el gen maestro de la lateralidad izquierda presente en la embriogénesis cardiaca. Sus alteraciones han condicionado anomalías de lateralidad como dextrocardia, defectos del tabique ventricular y auricular, doble salida del ventrículo derecho, defectos del tracto de salida como transposición de grandes vasos o estenosis pulmonar, y arritmias cardiacas como la fibrilación auricular. En particular, en un estudio se encontró una sustitución de adenina por guanina en la codificación del nucleótido 441 del gen PITX2c en un paciente con doble salida del ventrículo derecho en combinación con un defecto del tabique ventricular ${ }^{15}$.

La coexistencia de múltiples malformaciones cardiacas congénitas atípicas que producen una alteración importante en la fisiología cardiaca supone un reto terapéutico por la dificultad que representa el restablecimiento de una fisiología distinta que sea compatible con la vida. Sin embargo, en este paciente se realizó un abordaje individualizado y se logró de forma exitosa un cerclaje de la arteria pulmonar que proporcionará una calidad de vida aceptable hasta que pueda ser llevado a una corrección quirúrgica definitiva. Se presentaron limitaciones importantes en el seguimiento del paciente a largo plazo por ser habitante de un área rural.

\section{Conflicto de intereses}

Los autores declaran que no existe conflicto de Intereses.

\section{Financiamiento}

No se recibió financiamiento de ninguna institución.

\section{Responsabilidades éticas}

Protección de personas y animales. Los autores declaran que para esta investigación no se han realizado experimentos en seres humanos ni en animales.

Confidencialidad de los datos. Los autores declaran que han seguido los protocolos de su centro de trabajo sobre la publicación de datos de pacientes.

Derecho a la privacidad y consentimiento informado. Los autores han obtenido el consentimiento informado de los pacientes y/o sujetos referidos en el artículo. Este documento obra en poder del autor de correspondencia.

\section{Bibliografía}

\footnotetext{
1. Tassinari S, Martínez-Vernaza S, Erazo-Morera N, Pinzón-Arciniegas MC, Gracia G, Zarante I. Epidemiología de las cardiopatías congénitas en Bogotá, Colombia, en el período comprendido entre 2001 y 2014: ¿mejoría en la vigilancia 0 aumento en la prevalencia? Biomédica. 2018;380:141-8
} 
Rev Colomb Cardiol. 2021;28(4)

2. Walters HW, Mavroudis $\mathrm{C}$, Tchervenkov $\mathrm{Cl}$, Jacobs JP, Lacour-Gayet $\mathrm{F}$ Jacobs ML. Congenital Heart Surgery Nomenclature and Database Project: double outlet right ventricle. Ann Thorac Surg. 2000;69 (Suppl 1): S249-69.

3. Lev M, Bharati S, Meng CCL, Liberthson RR, Paul MH, Idriss F. A concept of double-outlet right ventricle. J Thorac Cardiovasc Surg. 1972;64:271-81.

4. Musumeci F, Shumway S, Lincoln C, Anderson RH. Surgical treatment for double-outlet right ventricle at the Brompton Hospital, 1973 to 1986. J Thorac Cardiovasc Surg. 1988;96:278-87.

5. Acherman RJ, Díaz G, Luna CF, Castillo W, Siassi B. Doble salida del ventrículo derecho. En: Díaz G, Sandoval N, Vélez JF, editores. Cardiología Pediátrica. 2018;2:621-34.

6. Muñoz-Castellanos L, Kuri M. Doble salida de ventrículo derecho. Enfoque embriológico. Arch Cardiol Mex. 2012;82:273-81.

7. Marino B, Versacci $P$, Guccione $P$, Carotti A. Malposiciones cardíacas En: Díaz G, Sandoval N, Vélez JF, editores. Cardiología Pediátrica. 2018;2:270-4

8. Díaz G, Díaz R. Hipertensión pulmonar secundaria. En: Díaz G, Sandoval N, Vélez JF, editores. Cardiología Pediátrica. 2018;2:82535 .
9. Egbe AC, Connolly HM, Miranda WR, Ammash NM, Hagler DJ, Veldtman GR, et al. Hemodynamics of Fontan failure: the role of pulmonary vascular disease. Circ Heart Fail. 2017;10: e004515.

10. Angeli E, Pace Napoleone C, Turci S, Oppido G, Gargiulo G. Pulmonary artery banding. Multimed Man Cardiothorac Surg. 2012;2012:mms010.

11. Takayama H, Sekiguchi A, Chikada M, Noma M, Ishizawa A, Takamoto S. Mortality of pulmonary artery banding in the current era: recent mortality of PA banding. Ann Thorac Surg. 2002;74:1219-24.

12. Bocks M, Boe B, Galantowicz M. Neonatal management of congenital heart disease. En: Martin R, Fanaroff A, Walsh M, editores. Fanaroff and Martin's Neonatal-Perinatal Medicine. $11^{\text {th }}$ ed. Cleveland, Ohio: Elsevier; 2019. p. 1393-414.

13. Formigari R, Michielon G, Digilio MC, Piacentini G, Carotti A, Giardini A, et al. Genetic syndromes and congenital heart defects: how is surgical management affected? Eur J Cardiothorac Surg. 2009;35:606-14.

14. Van Nisselrooij AEL, Lugthart MA, Clur SA, Linskens IH, Pajkrt E, Rammeloo LA, et al. The prevalence of genetic diagnoses in fetuses with severe congenital heart defects. Genet Med. 2020;22:1206-14.

15. Wang J, Xin Y, Xu W, Liu Z, Qiu X, Qu X, et al. Prevalence and spectrum of PITX2c mutations associated with congenital heart disease. DNA Cell Biol. 2013;32:708-16. 